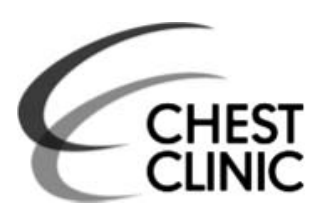
CHEST

${ }^{1}$ Research Department of Infection and Population Health, University College London, London, UK

${ }^{2}$ Tuberculosis Research Unit, National Heart and Lung Institute, Imperial College London, London, UK

\section{Correspondence to}

Professor Ajit Lalvani, Tuberculosis Research Unit, Imperial College London, London W21PG, UK; a. lalvani@imperial.ac.uk

Received 10 January 2013 Revised 10 January 2013 Accepted 11 January 2013

To cite: Abubakar I, Stagg HR, Whitworth $H$, et al. Thorax 2013;68: 298-301.

\title{
How should I interpret an interferon gamma release assay result for tuberculosis infection?
}

\author{
Ibrahim Abubakar, ${ }^{1}$ Helen Ruth Stagg, ${ }^{1}$ Hilary Whitworth, ${ }^{2}$ Ajit Lalvani ${ }^{2}$
}

\begin{abstract}
Background Interferon gamma release assays (IGRAs) are the first new diagnostic tests for latent tuberculosis (TB) infection (LTBI) since the century-old tuberculin skin test (TST). They are cell-mediated immune-based blood tests that have revolutionised LTBI diagnosis and are increasingly recommended by national guidelines.

Objectives With the rapid expansion of the IGRA evidence-base in recent years, the limitations of IGRA and uncertainties in clinical interpretation of IGRA results have increasingly come into focus. In LTBI diagnosis these include: prognostic power of IGRAs relative to TST for quantifying risk of progression to active disease, false-negative rates in immunocompromised patients, the clinical meaning of IGRA reversion and the significance of the size of IGRA response. Furthermore, the role of IGRAs in the diagnostic work-up of active TB is unclear, and there is little evidence supporting use of the tests in anti-TB treatment monitoring.

Methodological approach On-going large prospective longitudinal clinical endpoint cohort studies of active and latent TB will tackle some of the uncertainties regarding IGRAs. Here we discuss clinical practice and guidance in light of the current uncertainties, based on existing evidence.

Conclusions and impact Current and planned clinical research will fill the gaps in the evidence-base, narrowing the areas of uncertainty and informing future policy. Translational research into next-generation IGRAs and new T cell-based diagnostic platforms will likely overcome the limitations of current IGRAs in the near future.
\end{abstract}

\section{INTRODUCTION}

Mycobacterium tuberculosis (Mtb) causes almost 9 million cases of active tuberculosis (TB) annually, resulting in over 1.4 million deaths ${ }^{1}$; one-third of the world's population is believed to be latently infected. ${ }^{2}$ The incidence of TB in the UK has risen over the last two decades ${ }^{3}$ and, in line with most high-income countries, the majority of cases occur in foreign-born individuals from high-incidence nations.

Individuals with latent TB infection (LTBI) comprise a large reservoir from which future active cases arise, but chemoprophylactic treatment can effectively prevent subsequent development of active TB. Diagnosis and treatment of LTBI is therefore a cornerstone of TB control in high-income low-incidence countries. Since the risk of progressing to active $\mathrm{TB}$ is highest within the first 2-3 years following infection, ${ }^{4}$ this strategy specifically targets recently exposed individuals as well as those with risk factors for increased risk of progression to active $\mathrm{TB}$, in particular HIV-associated or iatrogenic immunosuppression.

Until recently the diagnosis of LTBI was dependent on the tuberculin skin test (TST), which suffers from poor specificity (due to confounding by prior BCG vaccination) and low sensitivity in immunocompromised patients. Interferon-gamma release assays (IGRAs) are blood tests developed to improve diagnosis by measuring the ex vivo cellular immune response to $M t b$-specific antigens, ${ }^{5}$ and are commercially available as QuantiFERON ${ }^{\circledR}$-TB Gold In-Tube (QFT-GIT) (Cellestis, Carnegie, Australia) and T-SPOT ${ }^{\circledR} . T B$ (Oxford Immunotec, Abingdon, UK). These tests are not confounded by prior BCG vaccination. A positive IGRA in someone with no clinical or radiological evidence of active TB thus constitutes a diagnosis of LTBI.

Published guidelines in most high-income lowincidence countries now recommend use of IGRAs in the diagnosis of $M t b$ infection, ${ }^{6}{ }^{7}$ either in a two-step testing strategy where the IGRA is used to confirm $M t b$ infection in BCG-vaccinated persons who test positive by TST or as a single-step IGRA. However, there are several uncertainties in their clinical application and interpretation (table 1).

\section{UNCERTAINTIES IN IGRA TESTING Latent TB}

The central challenge of treatment for LTBI is identifying those with the highest risk of progression. Since only approximately 5\% of TST-positive TB contacts progress to active TB within 2-5 years of exposure, ${ }^{4}$ the number of TST-positive contacts requiring treatment to prevent one case of active TB (number needed to treat, NNT) is about 20. Given that the TB burden in low-incidence countries is largely driven by LTBI progressing to active disease, a more powerfully predictive test to stratify latently infected individuals by progression risk would improve targeting of preventive treatment thereby reducing the NNT.

Several longitudinal studies have confirmed that IGRAs predict progression from LTBI to active TB. However, their prognostic power compared with the TST has been a key area of uncertainty. One systematic review of 15 studies with 26680 individuals concluded that the prognostic power of IGRAs and the TST were low and broadly equivalent ${ }^{8}$ while another review of 28 studies including $>30000$ individuals found IGRAs to be more predictive than the TST. ${ }^{9}$ Updated guidelines from the National Institute for Health and Clinical Excellence (NICE) on the use of IGRAs ${ }^{10}$ highlighted the paucity of high-quality studies of the 
Table 1 Challenges in interpreting IGRA results in various clinical settings

\begin{tabular}{|c|c|}
\hline $\begin{array}{l}\text { Subgroups considered for IGRA } \\
\text { testing by NICE }\end{array}$ & Uncertainties of IGRAs \\
\hline $\begin{array}{l}\text { New entrants from high incidence } \\
\text { countries }\end{array}$ & $\begin{array}{l}\text { Which immigrant subgroups to } \\
\text { target? }\end{array}$ \\
\hline Contacts of a TB outbreak & What is the meaning of reversion? \\
\hline Healthcare workers & \\
\hline Adult contacts of active TB cases & \\
\hline $\begin{array}{l}\text { Paediatric contacts of active TB } \\
\text { cases }^{*}\end{array}$ & $\begin{array}{l}\text { False negative rates unknown in } \\
\text { high-risk populations }\end{array}$ \\
\hline Immunocompromised patients* & \\
\hline Active TB† & False negative rates in $15-25 \%$ \\
\hline \multirow{2}{*}{$\begin{array}{l}\text { Active and latent TB: effect of } \\
\text { treatment } \dagger\end{array}$} & Increased risk of false negative results \\
\hline & Unsuitable for treatment monitoring \\
\hline \multicolumn{2}{|c|}{$\begin{array}{l}\text { *NICE recommends testing with both IGRA and the tuberculin skin test. } \\
\text { tNICE does not recommend IGRA testing but highlights potential uses which are the } \\
\text { subject of further study. } \\
\text { IGRA, interferon gamma release assay; NICE, National Institute for Health and Clinical } \\
\text { Excellence; TB, tuberculosis. }\end{array}$} \\
\hline
\end{tabular}

prognostic power of IGRAs relevant to low-incidence settings on which to base clinical recommendations.

A new study published in this issue of Thorax makes an important contribution to this evidence base and may help to inform IGRA-based screening policies. ${ }^{11}$ A single-step QFT-GIT screening strategy in 1769 recent TB contacts was evaluated in routine practice in Leicester, UK. Among untreated contacts, QFT-GIT had 70\% sensitivity and 86\% specificity for identifying LTBI progressing to active disease in 2 years, and the NNT of QFT-positive cases to prevent one case of active TB was 7, substantially lower than previously reported for the TST. This figure needs to be interpreted with caution, however, as it is lower than that reported by almost all previous studies and meta-analyses and may reflect inherent bias in the study design because the investigators making the diagnoses were not blinded to the IGRA results. Nonetheless, the study shows promise for the use of IGRAs in contact investigations to improve targeting of preventive therapy. However, IGRA results were not predictive of progression to disease in older contacts ( $\geq 36$ years), most likely due to a higher burden of infection that was remotely acquired (and therefore less likely to progress) in this mostly foreign-born population, analogous to the disappointing prognostic power of IGRAs in contacts in high-burden countries.

Available longitudinal outcome data and the higher specificity of IGRAs collectively suggest that the NNT for IGRA-positive contacts is lower than for TST-positive contacts, but most available evidence suggests it is still far from that required to effect a step change in TB control and prevention. Enhancing the prognostic power of IGRAs, thereby reducing the NNT, is therefore an urgent research priority (box 1).

Since recently infected contacts are at higher risk of progression to active $\mathrm{TB}$, a test able to distinguish recently acquired infection from remotely acquired infection would substantially reduce the NNT. Neither IGRAs nor the TST are capable of this discrimination, ${ }^{12}{ }^{13}$ and targeting recently infected persons will continue to depend on epidemiological risk factors until such a test is developed.

Some studies suggest that a larger size of baseline IGRA response may be predictive of progression, ${ }^{14}$ but there are currently insufficient data to use this clinically to risk-stratify, and more data correlating quantitative baseline responses with clinical
Box 1 Current National Institute for Health Research Health Technology Assessment (NIHR HTA)-funded research addressing interferon-gamma release assay (IGRA) uncertainties

\section{Latent TB: PREDICT}

Clinical uncertainty: Prognostic value of IGRA compared with the tuberculin skin test (TST) for predicting progression to active tuberculosis (TB)

Study design: Prospective longitudinal cohort

Population: New entrants and TB contacts

Intervention: Baseline IGRA

Outcome: Development of culture confirmed TB

Uncertainties addressed:

- Prognostic power of QFT-GIT vs T-SPOT ${ }^{\circledR}$.TB vs TST

- Prognostic power of IGRAs in high-risk populations including HIV-infected participants

- Prognostic power of quantitative baseline IGRA responses

- Clinical significance of IGRA reversion

- Improved prognostic power of next-generation IGRAs

\section{Active TB: IDEA}

Clinical uncertainty: Clinical utility of IGRA in diagnostic work-up of active TB

Study design: Prospective longitudinal cohort

Population: NHS patients with suspected active TB

Intervention: Baseline IGRA

Outcome: Final diagnosis of active TB

Uncertainties addressed:

- Role of QFT-GIT vs T-SPOT ${ }^{\circledR}$.TB vs TST

- If and where IGRAs fit in diagnostic algorithm for evaluation of suspected active TB

- Cost-effectiveness of diagnostic strategies

- Role of next-generation IGRAs in treatment monitoring

- Role of next-generation IGRAs in rapidly ruling out suspected active TB

- Role of next-generation IGRAs in treatment monitoring

outcomes are required (box 1). Therefore, results should currently only be interpreted in a binary fashion as positive or negative. Borderline results are uninterpretable and have no clinical significance, and should therefore be repeated. Indeterminate results, which usually result from a failed positive control and are more common in the setting of suppressed cellular immunity, are also uninterpretable and should also be repeated.

Where IGRAs are repeated, results may change from positive to negative or vice versa. ${ }^{15}{ }^{16}$ Conversion from negative to positive has similar clinical implications to TST conversion, reflecting recent acquisition of infection. The clinical significance of reversion from positive to negative is uncertain, although it may reflect spontaneous clearance of infection and low/absent risk of progression. Only longitudinal clinical outcome studies will determine if this is the case (box 1).

\section{Active TB}

IGRAs measure a cellular immune response to $M t b$-specific antigens. A positive result therefore indicates infection, but does not distinguish active from latent TB. Since $M t b$ infection is a prerequisite for TB disease, a negative IGRA result might provide a rapid 'rule-out' for active TB. However, this requires very high diagnostic sensitivity and, although IGRAs may have higher 
sensitivity than the TST, neither is sensitive enough to exclude a diagnosis of active TB. ${ }^{17} 18$

It is also proposed that a positive IGRA may provide a useful adjunct to 'rule-in' the diagnosis in paediatric patients with a clinical picture consistent with active TB, as the TST is already used. ${ }^{19}$ Furthermore, NICE guidelines suggest that TST/IGRA can be beneficial in diagnosing cases where histological appearances are not conclusive during the investigation of non-pulmonary TB. ${ }^{20}$ Clinical studies already indicate that next-generation IGRAs, incorporating additional $M t b$ antigens or measuring additional $M t b$-induced cytokines, provide significantly improved diagnostic sensitivity (without compromising specificity) that could enable rapid rule-out of suspected active TB (box 1). ${ }^{21} 17$ Newer T cellbased diagnostic platforms utilise $\mathrm{T}$ cell functional signatures that reflect mycobacterial burden to differentiate active from latent $\mathrm{TB}$ and provide treatment monitoring tools. ${ }^{22-24}$

\section{IS ONGOING RESEARCH LIKELY TO RESOLVE THE UNCERTAINTIES?}

The UK National Institute for Health Research Health Technology Assessment Programme (NIHR HTA) UK Prognostic Evaluation of Diagnostic IGRA ConsorTium (PREDICT) study is a large cohort study in which 10000 participants at high risk for LTBI (TB contacts and new entrants from high-incidence countries) are undergoing IGRA and TST and are then being followed up for 2-3 years to determine progression rates by baseline test result. A wide range of novel biomarkers, including nextgeneration IGRAs, are also being assessed to correlate baseline responses with clinical outcomes (box 1).

The NIHR HTA-funded IGRAs in Diagnostic Evaluation of Active TB (IDEA) study will assess the role of current and nextgeneration IGRAs in the diagnostic evaluation of 1012 patients prospectively presenting with suspected active TB in routine NHS practice (box 1). Evidence-based diagnostic algorithms and care pathways will be developed and health economic analyses performed to quantify the clinical utility and cost-effectiveness of current and next-generation IGRAs in the diagnostic assessment of suspected active TB.

Additional studies to quantify and compare the predictive value of IGRAs include a European TBNET study currently taking place using available routinely collected data in contacts (http://www.tb-net.org/content/view/22/53/) and a large prospective study, similar to PREDICT, planned by the US Centers for Disease Control and Prevention.

\section{WHAT SHOULD WE DO IN THE LIGHT OF UNCERTAINTY?}

The current NICE guidelines ${ }^{10} 20$ take account of most of the uncertainty around the prognostic power of existing IGRAs (table 1) and should be followed until other studies are completed. Given the paucity of evidence on the prognostic power of IGRAs in young children and immunocompromised individuals and the risk of false-negative results, concurrent IGRA and TST are recommended by NICE in children aged $<5$ years and HIV-positive individuals with a CD4 cell count of $<200$ cells $/ \mathrm{mm}^{3}$. IGRAs should not be used in the diagnosis of active TB until tests of higher sensitivity are available. ${ }^{5}$

\section{CURRENT AND FUTURE POLICY}

Given that $>75 \%$ of the UK's TB burden is carried by foreignborn immigrants, and most of this is due to reactivation of LTBI within the first several years after arrival, tackling imported LTBI in new entrants is increasingly recognised as an attractive strategy to reverse the relentless rise in UK TB rates. ${ }^{25}$ Policy is evolving rapidly in this area in response to a growing evidence base. For new entrant screening, NICE recommends using chest $\mathrm{x}$-rays and either the two-step TST with confirmatory IGRA or single-step IGRA. NICE recently substantially broadened its recommendations on which new entrants to screen from those who originate from sub-Saharan Africa to all those from countries with a TB incidence of $>40 / 100000$, which is lower than some urban centres in the UK. Moreover, the requisite resources to screen such a high proportion of all new entrants are not available; overstretched TB services in parts of the UK with the highest TB burdens often lack sufficient resources to screen any new entrants. ${ }^{26}$ However, an intermediate screening threshold of TB incidence in the country of origin to preferentially target migrant subgroups-such as those from the Indian subcontinent and sub-Saharan Africa who are most likely to have LTBI-is probably more cost-effective and pragmatic, as evidenced by a recent multicentre UK study. ${ }^{27}$

A study published in the current issue of Thorax provides further evidence to potentially inform policy on new entrant screening. ${ }^{28}$ First, it compared the cost-effectiveness of TST and each of the two IGRA platforms for new entrant screening and found that single-step testing is more cost-effective than two-step testing, particularly with QFT-GIT. Second, taken together with a previous new entrant screening study, ${ }^{29}$ it supports a recently proposed streamlined algorithm to new entrant LTBI screening, reserving chest radiography only for those testing positive by IGRA. ${ }^{25}$ This emerging approach, however, assumes that IGRA false-negative rates in contacts with asymptomatic active TB are very low, but more data are needed to support this. ${ }^{25}$ Third, it demonstrates that IGRA-based LTBI screening in immigrants can be reliably and cost-effectively carried out in primary care. ${ }^{28}$ However, due to variable levels of registration with general practitioners among migrants, ${ }^{30}$ implementation of IGRA screening should be accompanied by a drive to encourage access to primary care.

In conclusion, while several clinically important uncertainties remain, research is rapidly filling the gaps in the evidence base which, in turn, is informing policy and guiding practice.

Contributors All authors contributed to the conception and writing of this manuscript and have agreed on the final version of the paper. IA is guarantor

Funding IA and HRS are funded through a NIHR Senior Research Fellowship (grant number SRF-2011-04-001). AL is a Wellcome Trust Senior Research Fellow in Clinical Science and NIHR Senior Investigator. HSW is funded by an NIHR HTA program grant, IDEA (grant number P32907 164104).

Disclaimer The views expressed in this publication are those of the author(s) and not necessarily those of the NHS, the National Institute for Health Research or the Department of Health.

Competing interests $A L$ is inventor of several patents underpinning $T$ cell-based diagnosis, including the ESAT-6/CFP-10 ELISpot-based IGRA, T-SPOT ${ }^{\circledR}$. TB which was commercialised by a University of Oxford spin-out company, Oxford Immunotec Ltd, in which the University of Oxford and AL have minority shareholdings and royalty entitlements. AL's research is independent of Oxford Immunotec Ltd, which has no access to his research results and has never funded his research. All other authors declare no support from any organisation for the submitted work, no financial relationships with any organisations that might have an interest in the submitted work in the previous 3 years and no other relationships or activities that could appear to have influenced the submitted work.

Provenance and peer review Not commissioned; internally peer reviewed.

\section{REFERENCES}

1 World Health Organization (WHO). Global tuberculosis control 2011. Geneva: WHO 2012. http://www.who.int/tb/publications/global_report/en/index.html (accessed 25th January 2013).

2 Dye $\mathrm{C}$, Scheele S, Dolin P, et al. Consensus statement. Global burden of tuberculosis: estimated incidence, prevalence, and mortality by country. WHO Global Surveillance and Monitoring Project. JAMA 1999:282:677-86. 
3 Abubakar I, Lipman M, Anderson C, et al. Tuberculosis in the UK-time to regain control. BMJ 2011;343:d4281.

4 Ferebee SH. Controlled chemoprophylaxis trials in tuberculosis. A general review. Bibl Tuberc 1970;26:28-106.

5 Pai M, Riley LW, Colford JM. Interferon-gamma assays in the immunodiagnosis of tuberculosis: a systematic review. Lancet Infect Dis 2004;4:761-76.

6 National Institute for Health and Clinical Excellence (NICE). Clinical diagnosis and management of tuberculosis, and measures for its prevention and control: IGRA partial update. London: NICE2011.

7 Mazurek GH, Jereb J, Vernon A, et al. Updated guidelines for using Interferon Gamma Release Assays to detect Mycobacterium tuberculosis infection-United States, 2010. MMWR Recomm Rep 2010;59:1-25.

8 Rangaka MX, Wilkinson KA, Glynn JR, et al. Predictive value of interferon- $\gamma$ release assays for incident active tuberculosis: a systematic review and meta-analysis. Lancet Infect Dis 2012;12:45-55

9 Diel R, Loddenkemper R, Nienhaus A. Predictive value of interferon-gamma release assays and tuberculin skin testing for predicting progression from latent TB infection to disease state: a meta-analysis. Chest 2012;142:63-75.

10 Abubakar I, Griffiths C, Ormerod L. Diagnosis of active and latent tuberculosis: summary of updated NICE guidelines. BMJ (Clin Res Ed) 2012;345:e6828.

11 Haldar $\mathrm{P}$, Thuraisingam $\mathrm{H}$, Patel $\mathrm{H}$, et al. Single-step QuantiFERON screening of adult contacts: a prospective cohort study of tuberculosis risk. Thorax Published Online First: 6 September 2012. doi:10.1136/thoraxinl-2011-200956

12 Hinks TSC, Dosanjh DPS, Innes JA, et al. Frequencies of region of difference 1 antigen-specific but not purified protein derivative-specific gamma interferonsecreting T cells correlate with the presence of tuberculosis disease but do not distinguish recent from remote latent infections. Infect Immun 2009;77:5486-95.

13 Bradshaw $L$, Davies $E$, Devine $M$, et al. The role of the interferon gamma release assay in assessing recent tuberculosis transmission in a hospital incident. PLOS ONE. 2011;6:e20770.

14 Lienhardt C, Fielding K, Hane AA, et al. Evaluation of the prognostic value of IFN-gamma release assay and tuberculin skin test in household contacts of infectious tuberculosis cases in Senegal. PLoS ONE 2010;5:e10508.

15 Menzies D, Pai M, Comstock G. Meta-analysis: new tests for the diagnosis of latent tuberculosis infection: areas of uncertainty and recommendations for research. Ann Intern Med 2007:146:340-54

16 Pai $M$, Joshi R, Dogra $S$, et al. Serial testing of health care workers for tuberculosis using interferon-gamma assay. Am J Respir Crit Care Med 2006;174:349-55.

17 Dosanjh DP, Hinks TS, Innes JA, et al. Improved diagnostic evaluation of suspected tuberculosis. Ann Intern Med 2008;148:325-36.
18 Sester M, Sotgiu G, Lange $C$, et al. Interferon- $\gamma$ release assays for the diagnosis of active tuberculosis: a systematic review and meta-analysis. Eur Respir J 2011;37:100-11.

19 Mandalakas AM, Detjen AK, Hesseling AC, et al. Interferon-gamma release assays and childhood tuberculosis: systematic review and meta-analysis. Int I Tuberc Lung Dis 2011;15:1018-32.

20 National Institute for Health and Clinical Excellence. Tuberculosis: Clinical diagnosis and management of tuberculosis, and measures for its prevention and control. Clinical Guideline 117. London: NICE, 2011. http://guidance.nice.org.uk/CG117 (accessed 25th January 2013).

21 Millington KA, Fortune SM, Low J, et al. Rv3615c is a highly immunodominant RD1 (Region of Difference 1)-dependent secreted antigen specific for Mycobacterium tuberculosis infection. Proc Natl Acad Sci USA 2011;108:5730-5.

22 Casey R, Blumenkrantz D, Millington $\mathrm{K}$, et al. Enumeration of functional T-cell subsets by fluorescence-immunospot defines signatures of pathogen burden in tuberculosis. PLoS One 2010;5:e15619.

23 Harari A, Rozot V, Enders FB, et al. Dominant TNF- $\alpha+$ Mycobacterium tuberculosisspecific CD4+ T cell responses discriminate between latent infection and active disease. Nat Med 2011;17:372-6.

24 Whitworth HS, Scott M, Connell DW, et al. IGRAs - The gateway to T cell based TB diagnosis. Methods Published Online First: 3 Jan 2013. pii: S1046-2023(12) 00326-X

25 Lalvani A, Pareek M. Immigrant screening for TB: a missed opportunity to improve TB control in the United Kingdom. Pathog Glob Health 2012;106:5-7.

26 Pareek M, Abubakar I, White PJ, et al. UK immigrant screening is inversely related to regional tuberculosis burden. Thorax 2011;66:1010.

27 Pareek M, Watson JP, Ormerod LP, et al. Screening of immigrants in the UK for imported latent tuberculosis: a multicentre cohort study and cost-effectiveness analysis. Lancet Infect Dis 2011;11:435-44.

28 Pareek M, Bond M, Shorey J, et al. Community-based evaluation of immigrant tuberculosis screening using interferon $\gamma$ release assays and tuberculin skin testing: observational study and economic analysis. Published Online First: 12 June 2012. doi:10.1136/thoraxjnl-2011-201542

29 Hardy AB, Varma R, Collyns T, et al. Cost-effectiveness of the NICE guidelines for screening for latent tuberculosis infection: the QuantiFERON-TB Gold IGRA alone is more cost-effective for immigrants from high burden countries. Thorax 2010;65:178-80

30 Stagg HR, Jones J, Bickler $\mathrm{G}$, et al. Poor uptake of primary healthcare registration among recent entrants to the UK: a retrospective cohort study. BMJ Open 2012;2 